\title{
Cuestionario Honey-Alonso de Estilos de Aprendizaje: Nuevas evidencias psicométricas en población argentina
}

\author{
Agustín Freiberg-Hoffmann; Facundo Abal; Mercedes Fernández-Liporace
}

How to cite this article:

Freiberg-Hoffmann, A., Abal, F., \& Fernández-Liporace, M. (2020). Honey-Alonso Learning Styles Questionnaire: New psychometric evidences in Argentinean population. Acta Colombiana de Psicología, 23(2), 328-338. http://doi. org/10.14718/ACP.2020.23.2.13

Recibido, diciembre 23/2019; Concepto de evaluación, marzo 09/2019; Aceptado, marzo 17/2020

\author{
Agustín Freiberg-Hoffmann* \\ Universidad de Buenos Aires, CONICET, Buenos Aires, Argentina \\ ORCID: https://orcid.org/0000-0001-8737-1186 \\ Facundo Abal \\ Universidad de Buenos Aires, CONICET, Buenos Aires, Argentina \\ ORCID: https://orcid.org/0000-0001-7023-5380 \\ Mercedes Fernández-Liporace \\ Universidad de Buenos Aires, CONICET, Buenos Aires, Argentina \\ ORCID: https://orcid.org/0000-0001-7044-8386
}

\begin{abstract}
Resumen
En el presente trabajo se examinan las propiedades psicométricas de la versión argentina del Cuestionario Honey-Alonso de Estilos de Aprendizaje (CHAEA). Primero se analiza la estructura interna de la escala mediante un análisis factorial confirmatorio y de invarianza factorial, segmentando la muestra según facultad; luego se estudia la consistencia interna de las dimensiones confirmadas y se comparan los resultados con los obtenidos en estudios previos; posteriormente se examina la estabilidad temporal de las puntuaciones de cada dimensión; y, por último, se estudian las evidencias de validez concurrente con otra escala que evalúa enfoques de aprendizaje. Los resultados del análisis factorial confirmatorio muestran un adecuado ajuste del modelo testeado a partir de distintos métodos de estimación, mientras que con el análisis de invarianza factorial se comprueba la equivalencia métrica del modelo. Asimismo, los índices de consistencia interna son aceptables, aunque el análisis de estabilidad temporal de las dimensiones no comprueba diferencias significativas entre la primera y segunda administración del instrumento. Finalmente, en consonancia con hallazgos previos, las evidencias de validez concurrente exhiben asociaciones positivas entre los estilos de aprendizaje y el enfoque profundo, así como asociaciones negativas entre los estilos y el enfoque superficial. Al final se discuten los resultados a partir de antecedentes teóricos y empíricos.

Palabras clave: estilos de aprendizaje, CHAEA, validez, consistencia interna, estabilidad temporal, estudiantes universitarios.
\end{abstract}

\footnotetext{
Secretaría de Investigaciones, Facultad de Psicología (UBA), 2352, Gral. Juan Lavalle, Buenos Aires, Argentina (C1052AAA). Tel.: +54114952-5481. agustinfreiberg@gmail.com
} 


\title{
Honey-Alonso Learning Styles Questionnaire: New psychometric evidences in Argentinean population
}

\begin{abstract}
The study is aimed at analyzing psychometric features of the local version of the Honey-Alonso Learning Styles Questionnaire. First, the inner structure of the scale is examined by means of a confirmatory factor analysis and an analysis of factorial invariance, splitting the sample by faculty. Second, internal consistency coefficients of the dimensions are analyzed and compared to previous results. Third, external convergent validity evidences are considered, regarding scores obtained from a learning approach scale which was used as an external criterion. Findings show an adequate model fit, even employing different estimation methods. Furthermore, the factorial invariance analysis verifies the metrical equivalence of the model. The internal consistency study finds acceptable values. The dimensions' stability reliability indices do not verify significant differences between test and retest. Finally, the convergent validity evidences analyses show positive associations between learning styles and the Deep learning approach on the one hand, and negative associations between the Surface approach and learning styles on the other, as reported in previous studies. Results are discussed on the grounds of the theoretical and empirical background. Keywords: learning styles, CHAEA, validity, internal consistency, stability reliability, college students.
\end{abstract}

\section{Introducción}

Los estilos de aprendizaje se definen como las cualidades cognitivas, afectivas y fisiológicas que los estudiantes ponen en juego al momento de aprender (Keefe, 1982). Específicamente, el concepto apunta a describir diferencias individuales que se vinculan indirectamente con el rendimiento académico (Jiraporncharoen et al., 2015; Mozaffari et al., 2020; Wilkinson et al., 2014); de modo que estudiar y evaluar los estilos de aprendizaje permite formular ajustes en las estrategias de enseñanza según las particularidades individuales, en busca de mejores resultados en términos de calidad en los procesos de enseñanza-aprendizaje (Gutiérrez-Tapias, 2018).

Teniendo esto en cuenta, diversos autores se han interesado en el análisis de esta variable (p. ej., Biabani \& Izadpanah, 2019; Cea-Rodríguez et al., 2018; Halili et al., 2019; Olanipekun et al., 2020 ), razón por la cual se ha diseñado un gran número de instrumentos para evaluar el constructo en población universitaria (p. ej., Alonso et al., 1994; Dunn et al., 1982; Grasha \& Riechmann, 1975; Honey \& Mumford, 1986; Jackson, 2002; Kolb, 1976; Myers \& Myers, 1980; Renzulli \& Smith, 1978; Rezler \& Rezmovic, 1981; Schmeck et al., 1977; Vermunt, 1998; Witkin et al., 1971).

En particular, en cuanto a las versiones en español, el Cuestionario Honey-Alonso de Estilos de Aprendizaje (CHAEA) de Alonso et al. (1994) es, actualmente, una de las herramientas más difundidas (Escanero-Marcén et al., 2016; Juárez-Lugo et al., 2012); sin embargo, su adaptación en la versión para la población argentina, desarrollada por Freiberg-Hoffmann y Fernández-Liporace (2013), permite evaluar cuatro estilos de aprendizaje, de los cuales solo uno —el estilo pragmático - corresponde al modelo planteado por los autores del instrumento (Alonso et al., 1994), mientras que los tres restantes - los estilos asimilador, adaptador, y convergente - provienen del modelo propuesto por Kolb (1976).

Específicamente, en esta escala los estudiantes con predominio del estilo asimilador poseen gran capacidad de abstracción, por lo que son capaces de comprender e integrar fácilmente información de diversa naturaleza, a la vez que logran explicarla a otros con sencillez y claridad; asimismo, se enfocan más en la robustez teórica de las ideas que en su valor práctico, y en situaciones de aprendizaje prefieren la lectura, la interpretación de textos y la comparación de modelos teóricos (Kolb \& Kolb, 2005). Por otra parte, quienes presentan un estilo predominantemente adaptador tienen gran habilidad para transferir ideas a situaciones prácticas, sumado a que, dado su interés por participar de actividades novedosas y desafiantes, se guían por la intuición antes que por el razonamiento lógico, de modo que aprenden mejor mediante la experiencia concreta, la fijación de objetivos y el trabajo grupal (Kolb, 1984).

Por otra parte, los estudiantes con preeminencia del estilo convergente destacan por su destreza para imaginar distintas aplicaciones prácticas para una misma idea, de modo que participan con entusiasmo en la resolución de problemas y su aporte se centra, fundamentalmente, en las cuestiones técnicas de la situación a resolver; de hecho, prefieren articular ideas nuevas y realizar simulaciones, pruebas en laboratorios y actividades con aplicación en situaciones reales (Kolb \& Kolb, 2005). Y, por último, los alumnos con predominio del estilo pragmático se caracterizan por su capacidad para el análisis conceptual y para la identificación de los aspectos que integran esos 
330

conceptos y su posible aplicación en la práctica con efectos positivos; además, son personas impacientes, seguras de sí y que gustan de actuar rápidamente cuando alguna idea les resulta interesante, de modo que en situaciones de aprendizaje se enfocan en la planificación de actividades prácticas, como lo son el análisis del material de estudio, la fijación de metas y la organización del tiempo; de hecho, se interesan en mayor medida por la utilidad de los contenidos que deben aprender (Alonso et al., 1994).

Ahora bien, el CHAEA se emplea habitualmente para describir perfiles estudiantiles individuales y grupales, así como para analizar la relación entre los distintos estilos de aprendizaje y el rendimiento académico, o para examinar diferencias entre alumnos que cursan diferentes disciplinas y que transitan diversos momentos en su trayectoria académica; razones por las cuales, según diversos investigadores, resulta una herramienta fundamental en las aulas universitarias (Altamirano-Droguett et al., 2019; Cardozo et al., 2018; Escanero-Marcén et al., 2018; Freiberg-Hoffmann et al., 2017; Ponce-Cumbreras \& Gamarra-Bustillos, 2015; PrietoLoureiro, 2019; Rodríguez et al., 2015; Villalba, 2015).

Sin embargo, a pesar de su uso extendido, las evidencias sobre su estructura interna son controversiales, por lo que la replicación de la solución factorial propuesta por los autores originales es, aún, objeto de debate (Lopes da Silveira, 2013; Orellana et al., 2002; Rodríguez-Gómez, 2006; Silva-Falchetti, 2009). De hecho, como se mencionó anteriormente, entre los estudios que analizan ese problema se encuentra la adaptación argentina del CHAEA(FreibergHoffmann \& Fernández-Liporace, 2013), cuya estructura no coincide completamente con la hipotetizada por Alonso et al. (1994), pues informa de la extracción y confirmación de cuatro dimensiones con adecuado ajuste, con puntajes aceptables de consistencia interna, congruentes con el número de reactivos conservados en cada dimensión, pero diferentes a los reportados en la escala original.

En virtud de los estos hallazgos, en la presente investigación se propuso analizar nuevas evidencias de validez y confiabilidad para el CHAEA en población universitaria local, con el fin de que los hallazgos se sumen e, incluso, amplíen lo encontrado en estudios psicométricos previos sobre la escala (Freiberg-Hoffmann \& Fernández-Liporace, 2013). De esta manera, como objetivos del presente estudio se planteó: (a) analizar la estructura interna del CHAEA — por medio de un análisis factorial confirmatorio, del análisis de invarianza factorial y del estudio de la consistencia interna de la escala—, (b) examinar la estabilidad temporal de sus puntuaciones, y (c) estudiar evidencias de validez concurrente con diferentes enfoques de aprendizaje.

\section{Método}

\section{Diseño}

Se realizó un estudio instrumental, de tipo transversal.

\section{Participantes}

Se empleó un muestreo por conveniencia, en el que se hizo uso de tres submuestras según el tipo de análisis que se pretendía realizar. De esta manera, para el análisis de la estructura interna del CHAEA se trabajó con 1342 estudiantes universitarios de Buenos Aires (43.7 \% varones y $56.3 \%$ mujeres) de distintas facultades $(31.8 \%$ de Psicología, $25.1 \%$ de Ciencias Exactas y Naturales, $21.4 \%$ de Ingeniería, $11.9 \%$ de Medicina, y $9.8 \%$ de Filosofía), de entre 17 y 36 años de edad $\left(M_{\text {edad }}=23.32 ; D E=2.82\right)$; mientras que para el estudio de la confiabilidad test-retest participaron 20 estudiantes de Psicología (20\% varones y $80 \%$ mujeres $)$ de entre 21 y 36 años $\left(M_{\text {edad }}=24.85\right.$; $D E=4.17)$; y para el análisis de evidencias de validez concurrente se trabajó con 789 estudiantes universitarios de Buenos Aires (48.7 \% varones y $51.3 \%$ mujeres) de 17 a 36 años $\left(M_{\text {edad }}=22.96 ; D E=3.68\right)$, provenientes de distintas facultades (40.6\% de Psicología, $27.9 \%$ de Ingeniería, $15.2 \%$ de Ciencias Exactas y Naturales, $8.9 \%$ de Derecho, y $7.5 \%$ de Medicina).

\section{Instrumentos}

Para la presente investigación se solicitó información sobre el género, edad y facultad de cada uno de los participantes del estudio, y se aplicó el Cuestionario HoneyAlonso de Estilos de Aprendizaje (CHAEA) y el Revised Two Factor Study Process Questionnaire (R-SPQ-2F).

Cuestionario Honey-Alonso de Estilos de Aprendizaje

Se utilizó la versión argentina para estudiantes universitarios desarrollada por Freiberg-Hoffmann y FernándezLiporace (2013) — descrita en la introducción—, compuesta por de 28 reactivos con respuesta dicotómica que evalúan cuatro estilos de aprendizaje - asimilador, convergente, adaptador y pragmático-.

Revised two factor study process questionnaire $(R-S P Q-2 F)$

Este instrumento, elaborado por Biggs et al. (2001), evalúa los enfoques de aprendizaje, definidos como los motivos y estrategias - moldeados por el contexto educativo-que emplean los estudiantes para procesar la información (Biggs, 1988). Desde este modelo destacan dos tipos de enfoques: 
uno profundo, propio de quienes se interesan por comprender los contenidos y por articularlos con conocimientos previos —asociado positivamente con el rendimiento académico-; y otro superficial, característico de alumnos con poco interés por el aprendizaje, que prefieren memorizar y reproducir sin comprender - asociado negativamente con el rendimiento académico-(Biggs \& Tang, 2011).

Con el fin de evaluar los enfoques de aprendizaje en esta investigación, se aplicó el cuestionario adaptado a estudiantes universitarios de Buenos Aires — adaptación realizada por Freiberg-Hoffmann y Fernández-Liporace (2016)—, que cuenta con 20 afirmaciones que se responden - por medio de una escala tipo Likert de cinco alternativas - según el grado de acuerdo del evaluado con cada una de ellas. Esta versión presenta una adecuada consistencia interna en sus dimensiones (alfas ordinales $>.80$ ), así como apropiadas evidencias de validez de constructo —análisis de componentes principales y análisis factorial confirmatorio$\mathrm{y}$ de validez de contenido y aparente - juicio experto y estudio piloto-.

En el presente estudio, además, se analizaron las propiedades psicométricas del instrumento a partir de la muestra seleccionada, para lo cual se aplicó un análisis factorial confirmatorio en el que se comprobó el ajuste del modelo a los datos empíricos — procedimiento que arrojó adecuados índices de ajuste CFI (.932), NFI (.915), NNFI (.924) y RMSEA (.066) - Posteriormente, se examinó la consistencia interna mediante el coeficiente alfa ordinal, con lo cual se obtuvieron valores aceptables en las dimensiones profundo (.755) y superficial (.839).

\section{Procedimiento}

Se recogieron los datos durante el horario de clases, para lo cual un psicólogo habilitado y debidamente entrenado se encargó de la aplicación de los instrumentos. Para la toma de los datos se informó a los estudiantes sobre el carácter voluntario y anónimo de su participación, y se les ofreció la posibilidad de cesar su respuesta en cualquier instancia de la aplicación. Además, los examinados firmaron un consentimiento informado en el que se explicitaban los objetivos del estudio y se aseguraba el tratamiento confidencial de resultados, así como el anonimato de los datos personales.

Análisis de datos

\section{Análisis de la estructura interna del CHAEA}

Para esta parte del análisis se empleó el software LISREL 8.8. Como primer paso, se examinó el supuesto de normalidad multivariante de las variables analizadas, $\mathrm{y}$, debido al incumplimiento de este supuesto, se decidió emplear métodos de estimación robustos (Holgado-Tello et al., 2018). De este modo, se aplicó el cálculo de matrices de correlaciones tetracóricas y el método de estimación de mínimos cuadrados ponderados (WLS), con lo cual se replicaba el proceder empleado en el estudio previo desarrollado por Freiberg-Hoffmann y Fernández-Liporace (2013).

Adicionalmente, se emplearon otros métodos de estimación —mínimos cuadrados ponderados diagonales (DWLS) y mínimos cuadrados no ponderados robusto (RULS) para analizar la independencia del modelo respecto del tipo de estimación empleado; y el ajuste y la parsimonia del modelo se interpretaron a partir de los mismos índices utilizados en el estudio precedente, antes citado, para facilitar la comparación de resultados —es decir, el goodness of fit index (GFI), el adjusted goodness of fit index (AGFI), el root mean square error of approximation (RMSEA), el parsimony goodness of fit index (PGFI) y el parsimony normed fit index (PNFI)-.

Por otro lado, para el análisis de la invarianza factorial se seleccionó la variable facultad para examinar si el instrumento funcionaba de la misma manera en estudiantes de distintas orientaciones académicas. Para esto, se testearon distintos modelos anidados, imponiendo diferentes niveles de restricción, de modo que, primero, se analizó un modelo configural - Modelo 1-, sin restricciones; luego, un modelo métrico -Modelo 2-, restringiendo los coeficientes de regresión; $\mathrm{y}$, por último, un modelo estructural -Modelo 3 -, restringiendo las covarianzas. Posteriormente, se aplicó el método de estimación de mínimos cuadrados no ponderados robusto (RULS), estadístico aconsejable para el tratamiento de variables categóricas y muestras pequeñas, que permite reducir el error Tipo I e incrementar la potencia estadística de los análisis (Holgado-Tello et al., 2018).

Por último, la invarianza se interpretó a partir de los índices CFI y RMSEA, aconsejados para el análisis de variables categóricas y muestras múltiples con distintos tamaños (Chen, 2007; Rojas et al., 2018); para el análisis de consistencia interna de las dimensiones se estimaron los índices $K R-20$ para las dimensiones de la escala; y, finalmente, se hicieron comparaciones entre el análisis actual y el estudio previo por medio del coeficiente $W$ de concordancia de Kendall (Feldt, 1969).

\section{Estudio de la confiabilidad test-retest}

Para este análisis se empleó el programa SPSS, v. 21, donde se analizó previamente el supuesto de normalidad —el cual resultó comprobado_; se estudió la estabilidad temporal de las puntuaciones de las dimensiones del cuestionario mediante un procedimiento paramétrico de medidas repetidas, con un intervalo de 30 días — prueba 
$t$ de Student para muestras relacionadas-; y se examinó, además, la asociación entre las puntuaciones pre y post-test por medio del coeficiente de correlación intraclase (ICC).

Análisis de evidencias de validez concurrente

Finalmente, para el análisis de la validez concurrente se utilizó el software SPSS, v. 21, con el cual se examinó el supuesto de normalidad de los datos — que no pudo ser verificado-; y se empleó una prueba no paramétrica para calcular el coeficiente de correlación rho de Spearman entre los estilos de aprendizaje del CHAEA y los enfoques de aprendizaje del R-SPQ-2F.

\section{Resultados}

Análisis de la estructura interna del CHAEA

\section{Análisis factorial confirmatorio}

Inicialmente, se calculó un análisis factorial confirmatorio con el fin de testear si el modelo factorial derivado del estudio previo de validación argentina de FreibergHoffmann y Fernández-Liporace (2013) se replicaba en la nueva muestra (véase Figura 1), y se encontró un adecuado ajuste de los resultados para todos los modelos testeados a partir de distintos métodos de estimación (véase Tabla 1).
Análisis de consistencia interna

Posteriormente, para el análisis de la consistencia interna de las dimensiones se encontraron coeficientes $K R-20$ aceptables para todas las dimensiones, y, en la comparación entre esos índices, obtenidos en ambos estudios locales — previo y actual-, se hallaron diferencias significativas $(p<.05)$ para los estilos asimilador y adaptador (véase Tabla 2).

\section{Análisis de invarianza factorial}

Finalmente, se analizó la invarianza del modelo aplicando distintos niveles de restricción; donde, en todos los casos, se comprobó la invariabilidad del modelo (véase Tabla 3).

Tabla 3.

CHAEA, invarianza factorial según facultad

\begin{tabular}{ccccc}
\hline & RMSEA [IC 90\%] & $\Delta$ RMSEA & CFI & $\Delta$ CFI \\
\hline Configural & $.061[.058-.064]$ & - & .969 & - \\
Métrico & $.061[.058-.063]$ & .000 & .967 & .002 \\
Estructural & $.061[.058-.063]$ & .000 & .967 & .002 \\
\hline
\end{tabular}

Nota. $* * p<.01$.

\section{Estudio de confiabilidad test-retest}

Con respecto a la confiabilidad del instrumento, se analizó la estabilidad temporal de sus dimensiones por medio de la comparación entre las puntuaciones obtenidas en dos momentos diferentes. Como resultado, no se observaron diferencias significativas (véase Tabla 4).

Tabla 1.

CHAEA. Índices de ajuste de modelos

\begin{tabular}{|c|c|c|c|c|c|c|c|}
\hline & \multicolumn{5}{|c|}{ Índices de ajuste } & \multicolumn{2}{|c|}{$\begin{array}{l}\text { Índices de } \\
\text { parsimonia }\end{array}$} \\
\hline & $\chi^{2}$ & $\mathrm{gl}$ & GFI & AGFI & RMSEA[IC]* & PGFI & PNFI \\
\hline $\begin{array}{l}\text { Primer estudio (Freiberg-Hoffmann \& } \\
\text { Fernández-Liporace, 2013) (WLS) }\end{array}$ & $1668.341 * *$ & 344 & .934 & .922 & $.075[.071-.078]$ & .792 & .669 \\
\hline Estudio actual (WLS) & $2126.880 * *$ & 344 & .960 & .953 & $.064[.059-.064]$ & .814 & .790 \\
\hline Estudio actual (DWLS) & $9841.310 * *$ & 344 & .949 & .939 & $.055[.053-.058]$ & .804 & .882 \\
\hline Estudio actual (RULS) & $10011.903^{* *}$ & 344 & .919 & .904 & $.055[.052-.057]$ & .779 & .882 \\
\hline
\end{tabular}

Tabla 2.

CHAEA, comparación de índices de consistencia interna

\begin{tabular}{ccccc}
\hline & Asimilador & Convergente & Adaptador & Pragmático \\
\hline Primer estudio (Freiberg-Hoffmann \& & .65 & .63 & .62 & .50 \\
Fernández-Liporace, 2013) (KR-20) & .70 & .60 & .72 & .49 \\
Estudio actual (KR-20) & .85 & .92 & .73 & .98 \\
W $_{(\text {KR-20, Actual - KR-20, 2013) }}$ & .01 & .12 & .00 \\
$\mathrm{p}_{(\text {KR-20, Actual - KR-20, 2013) }}$ & .38 \\
\hline
\end{tabular}




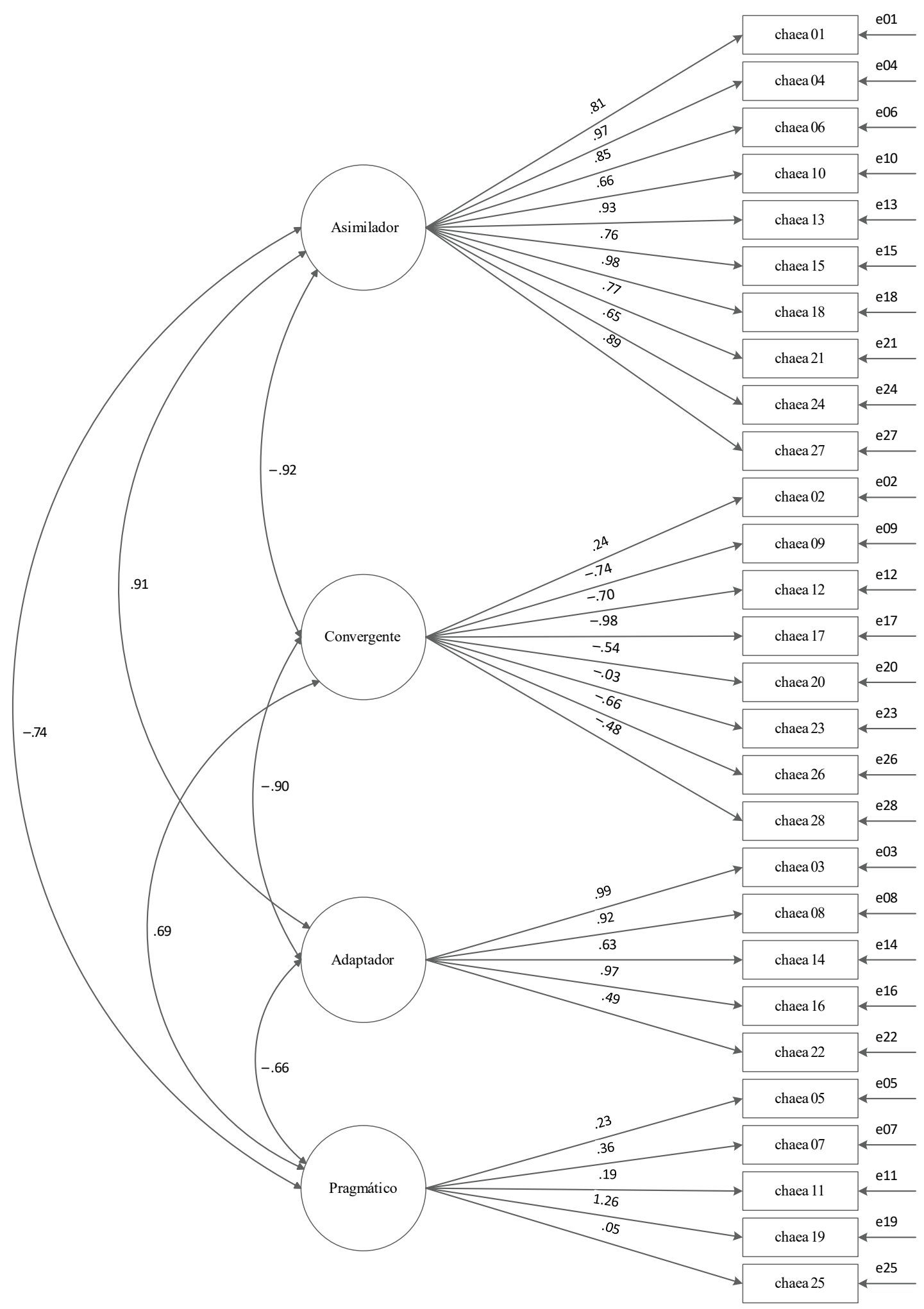

Figura 1. CHAEA, modelo tetrafactorial con el método de estimación WLS. 
Tabla 4.

CHAEA, estabilidad temporal de las puntuaciones de las dimensiones

\begin{tabular}{ccccc}
\hline Dimensión & $I C C$ & $t$ & $g l$ & $p$ \\
\hline Asimilador & $.783^{* *}$ & 1.453 & 19 & .163 \\
Convergente & $.850^{* *}$ & -.679 & 19 & .505 \\
Adaptador & $.834^{* *}$ & -.900 & 19 & .379 \\
Pragmático & $.507^{*}$ & .777 & 19 & .447 \\
\hline
\end{tabular}

Nota. ${ }^{* *} p<.01 ; * p<.05$.

Análisis de evidencias de validez concurrente

Por último, se correlacionaron las medidas de los estilos de aprendizaje (CHAEA) con las de los enfoques de aprendizaje (R-SPQ-2F) con el fin de obtener evidencias de validez concurrente. En este caso, se hallaron índices estadísticamente significativos en todos los casos, excepto para la asociación entre el estilo adaptador y el enfoque superficial (véase Tabla 5).

\section{Discusión}

El presente estudio aporta nuevos análisis con respecto a las propiedades psicométricas de la versión argentina del CHAEA para estudiantes universitarios. Específicamente, se llevó a cabo un análisis factorial confirmatorio y un estudio de consistencia interna con el fin de sumar evidencias y comparar sus resultados con los obtenidos en un estudio previo realizado sobre la misma población (Freiberg-Hoffmann \& Fernández-Liporace, 2013). Además, se examinaron nuevas evidencias de validez de constructo —análisis de invarianza factorial-, de criterio validez concurrente, y de confiabilidad en términos de estabilidad temporal. Desde un punto de vista aplicado, la investigación buscó aportar nuevos resultados sobre la calidad de la escala, para así otorgar a los usuarios información reciente sobre sus propiedades técnicas. A la vez, desde un enfoque conceptual, el estudio estuvo orientado a profundizar e incrementar evidencias sobre la estructura dimensional del constructo.

De este modo, por una parte, el análisis de la estructura interna del CHAEA confirmó un adecuado ajuste del modelo teórico a los datos empíricos, pues los índices de ajuste, obtenidos a partir de la aplicación de todos los métodos de estimación sugeridos para el tratamiento de variables categóricas - WLS, DWLS y RULS - (Koğar \& Koğar, 2015), fueron similares a los del estudio previo (FreibergHoffmann \& Fernández-Liporace, 2013). De hecho, en todos los casos se alcanzaron los valores sugeridos tanto para el GFI y el AGFI — superiores a .90 - como para el RMSEA -inferiores a .08- (Schumacker \& Lomax, 2016); resultados favorables para la hipótesis de robustez del modelo testeado, pues cada método produce siempre estimaciones sesgadas a causa de diversos factores - como el tamaño de la muestra, el número de variables observadas y latentes, y la cantidad de parámetros a estimar o de categorías de respuesta de las variables observadas, entre otras (Li, 2015)- A partir de esto, es posible hipotetizar que la estructura interna del instrumento no se vería afectada de modo significativo por el error de medición, lo que implica una mejor estimación de la puntuación verdadera.

Por otra parte, se encontró que el ajuste del modelo - estimado en el estudio actual mediante el método WLS - fue superior al hallado en el trabajo precedente - calculado con la misma metodología- (FreibergHoffmann \& Fernández-Liporace, 2013); diferencia que podría residir, probablemente, en el hecho de que la robustez de las estimaciones del método WLS depende, en principio, del tamaño muestral (Holgado-Tello et al., 2018; Jackson et al., 2009). Así, resulta esperable que en el análisis de la muestra actual $(N=1342)$, que duplica en tamaño a la del estudio previo $(N=682)$, se obtuvieran, por reducción del error de Tipo I, valores más elevados en los índices de ajuste. Esto permite justificar la diferencia de ajuste entre ambos modelos e interpretar los resultados hallados en ambos estudios de modo equivalente.

Por otro lado, con respecto a los parámetros estimados, si bien se observan algunos valores negativos, se ha decidido no aplicar algún tratamiento estadístico especial, dado que la ocurrencia de dichos valores es esperable cuando se trabaja con matrices de correlaciones tetracóricas, métodos de estimación libre asintótica y muestras heterogéneas (Yuan et al., 2011). Además, se ha establecido que la presencia

Tabla 5.

CHAEA, análisis de evidencias de validez concurrente con el R-SPQ-2F

\begin{tabular}{ccccc}
\hline \multirow{2}{*}{ Enfoques } & \multicolumn{4}{c}{ Estilos de aprendizaje } \\
\cline { 2 - 4 } & Asimilador & Convergente & Adaptador & Pragmático \\
\hline Profundo & $.294^{* *}$ & $.354^{* *}$ & $.183^{* *}$ & $.317^{* *}$ \\
Superficial & $-.180^{* *}$ & $-.304^{* *}$ & -.066 & $-.186^{* *}$ \\
\hline
\end{tabular}

Nota. $* * p<.01$. 
de esta clase de ecuaciones infractoras no tiene efectos significativos sobre estadísticos como el chi cuadrado (Chen et al., 2001).

Ahora bien, con respecto a la comparación de los índices de consistencia interna $(K R-20)$ entre el análisis actual y el trabajo previo (Freiberg-Hoffmann \& Fernández-Liporace, 2013), se observaron diferencias importantes entre los índices de las dimensiones adaptador y asimilador. En este punto es importante tener en cuenta que los coeficientes de consistencia interna son sensibles al tamaño muestral, dado que incrementa su valor al aumentar el número de sujetos (Feldt et al., 1987). Por tal motivo, parece razonable hipotetizar que las diferencias encontradas a favor del estudio aquí presentado serían atribuibles al mayor tamaño muestral antes que a diferencias reales en las puntuaciones observadas.

Por otra parte, respecto al análisis de invarianza factorial interfacultades, puede decirse que este nuevo estudio confirma la equivalencia de la estructura inicial del CHAEA. Esto implica que el instrumento no presenta un funcionamiento diferencial según la disciplina académica, lo que aporta evidencias favorables para la generalización del modelo. En otras palabras, las puntuaciones del CHAEA presentan una baja probabilidad de verse influidas por el sesgo de medida atribuible al modo distintivo en que los estudiantes de las distintas especialidades aquí contempladas responden a la prueba (Jak, 2014).

Adicional a esto, para concluir con el examen de la estructura interna debe destacarse la adecuada parsimonia del modelo, que pudo establecerse a partir de la interpretación de varios índices de ajuste, puesto que para todos los modelos estimados se reportaron valores RMSEA inferiores a .08, AGFI superiores a .90, y PNFI y PGFI superiores a .50 (Brown, 2015; Hahs-Vaughn, 2017; Mulaik, 1989). En términos aplicados, ello implica que el instrumento permite una evaluación de los estilos de aprendizaje a partir de las cuatro dimensiones estudiadas asimilador, convergente, adaptador y pragmático - que guardan una adecuada representatividad respecto al constructo, sin perder capacidad explicativa.

De otro lado, el examen de la estabilidad temporal de las dimensiones muestra que las puntuaciones de los estilos no variaron significativamente en un lapso de 30 días, lo cual da cuenta de que las fuentes de error que se experimentan con los cambios temporales - como el aprendizaje, el estado de ánimo o el estado físico, entre otras - no afectarían las medidas derivadas del instrumento durante dicho intervalo (Irwin \& Hughes, 2018).

Asimismo, con respecto al resultado del análisis de evidencias de validez concurrente, se observa que todos los estilos de aprendizaje se asociaron positivamente con el enfoque profundo y negativamente con el superficial; siendo una única excepción la no correlación entre el enfoque superficial y el estilo adaptador; no obstante, este estilo correlaciona positivamente con el enfoque profundo, con lo cual se comprueba la validez del estilo adaptador de modo parcial.

Estas asociaciones positivas y negativas con los enfoques profundo y superficial, respectivamente, resultan esperables dado que los estilos describen características estudiantiles asociadas a diferentes tipos de procesamiento de información que se manifiestan en situaciones de aprendizaje - características que incluyen distintos tipos de intereses y motivaciones representados en la noción de enfoques de aprendizaje (Biggs \& Tang, 2011)—. Además, cabe destacar que este resultado se orienta en la misma dirección que un estudio previo, en el que se reporta, a partir de un análisis factorial exploratorio, cómo dimensiones de los estilos se agrupan con el enfoque profundo y se asocian negativamente con el superficial (Cano-García \& Justicia-Justicia, 1994).

Con respecto a las bajas asociaciones encontradas, estas pueden explicarse por las diferencias entre los constructos analizados, ya que si bien ambos conceptos se relacionan teóricamente, cada uno de ellos se centra en aspectos diferenciales. Así, los estilos de aprendizaje examinarían las autopercepciones de los estudiantes sobre sus características cognitivas, afectivas y fisiológicas para aprender, mientras que los enfoques de aprendizaje, en cambio, indagarían la motivación y las estrategias que los educandos emplean como resultado de cómo perciben el contexto de enseñanza. Adicionalmente, cabe destacar que la ocurrencia de bajas asociaciones es esperable en el análisis de muestras amplias y heterogéneas (Ranganathan \& Aggarwal, 2016).

Finalmente, este trabajo presenta algunas limitaciones que deben tenerse en cuenta, como que la muestra empleada no contempla la totalidad de facultades que integran el sistema universitario de Buenos Aires; o que hizo falta un análisis de evidencias de validez predictiva que tome como criterio el rendimiento académico o la calidad de los aprendizajes logrados. Estas cuestiones deberán contemplarse en nuevos estudios, para que en ellos se recolecten datos sobre muestras más heterogéneas y se incluyan indicadores de rendimiento estudiantil, como, por ejemplo, el promedio de las calificaciones obtenidas, o índices complejos de calidad de aprendizaje.

Para concluir, se puede afirmar que con la presente investigación se obtuvieron hallazgos congruentes con la estructura dimensional reportada en la primera adaptación argentina de la escala que ofrecen nueva evidencia en favor de la generalización de este modelo — testeado en estudiantes universitarios de Buenos Aires-. Así, es posible concluir que no solo se confirman casi en su totalidad las evidencias 
336

reportadas para la versión argentina original, sino que, además, se incorporan nuevos aportes. Estos resultados, de cara al ámbito de aplicación, ofrecen a los profesionales del ámbito educativo una mayor certeza y confianza en las medidas obtenidas por medio del CHAEA. De esta manera, los usuarios pueden, a partir de la información arrojada por el cuestionario, tomar decisiones orientadas a mejorar los aprendizajes universitarios. Incluso, los resultados encontrados podrían ser útiles a la hora de planear talleres con objetivos determinados, cursos de capacitación para docentes según necesidades detectadas, modificaciones en los programas de las asignaturas, o bien cambios académicos mayores, como, por ejemplo, la actualización de los planes de estudio y la introducción de nuevas metodologías de enseñanza.

\section{Referencias}

Alonso, C. M., Gallego, D. J., \& Honey, P. (1994). Los estilos de aprendizaje. Procedimientos de diagnóstico y mejora. Mensajero.

Altamirano-Droguett, J., Araya-Crisóstomo, S., \& PazContreras, M. (2019). Learning styles and academic performance of Obstetrics' students. Revista Ciencias de la Salud, 17(2), 276-292. https://doi.org/10.12804/revistas.urosario. edu.co/revsalud/a.7937

Biabani, M., \& Izadpanah, S. (2019). The study of relationship between Kolb's learning styles, gender and learning American slang by Iranian EFL students. International Journal of Instruction, 12(2), 517-538. https://doi. org/10.29333/iji.2019.12233a

Biggs, J. B. (1988). Assessing student approaches to learning. Australian Psychologist, 23(2), 197-206. https://doi. org $/ 10.1080 / 00050068808255604$

Biggs, J. B., Kember, D., \& Leung, D. Y. (2001). The Revised Two Factor Study Process Questionnaire: R-SPQ-2F. British Journal of Educational Psychology, 71, 133-149. https://doi.org/10.1348/000709901158433

Biggs, J., \& Tang, C. (2011). Teaching for quality learning at university. McGrawHill.

Brown, T. (2015). Confirmatory factor analysis for applied research. Guilford Press.

Cano-García, F., \& Justicia-Justicia, F. (1994). Learning strategies, styles and approaches: an analysis of their interrelationships. Higher Education, 27, 239-260. https://doi. org/10.1007/bf01384091

Cardozo, L., Molano-Sotelo, E., Moreno-Jiménez, J., VeraRivera, D., \& Peña-Vega, M. (2018). Identificación de los estilos de aprendizaje: Estudiantes universitarios de entrenamiento deportivo de jornadas diurna y nocturna. Educación Física y Ciencia, 20(4), e060. https://doi. org/10.24215/23142561e 060
Cea-Rodríguez, J., Sanhueza-Burgos, H., \& Filgueira-Muñoz, E. (2018). Psychological types and learning styles of undergraduate students of an economic and administrative science faculty in Chile. Revista Academia y Negocios, 4(1), 65-80. http://ran.udec.cl/ojs/index.php/ran/article/view/60/97

Chen, F. F. (2007). Sensitivity of goodness of fit indexes to lack of measurement invariance. Structural equation modeling: A multidisciplinary Journal, 14(3), 464-504. https://doi. org/10.1080/10705510701301834

Chen, F., Bollen, K., Paxton, P., Curran, P., \& Kirby, J. (2001). Improper solutions in structural equation models. Sociological, Methods \& Research, 29(4), 468-508. https:// doi.org/10.1177/0049124101029004003

Dunn, R., Dunn, K., \& Price, G. (1982). Productivity Environmental Preference Survey. Price Systems.

Escanero-Marcén, J., Soria, M., \& Guerra-Sánchez, M. (2018). Estilos de aprendizaje y rendimiento académico: diferentes herramientas, diferentes resultados. Fundación Educación Médica, 21(4), 173-180. https://doi.org/10.33588/fem.214 .954

Escanero-Marcén, J., Soria, M., Guerra-Sánchez, M., \& Silva, J. (2016). Comparación de los estilos de aprendizaje de los alumnos de medicina obtenidos con un nuevo cuestionario con los proporcionados por el Cuestionario Honey-Alonso (CHAEA). Fundación Educación Médica, 19(1), 19-26. https://doi.org/10.33588/fem.191.819

Feldt, L. S. (1969). A test of the hypothesis that Cronbach alpha or Kuder-Richardson coefficient twenty is the same for two test. Psychometrica, 34(3), 363-373. https://doi. org $/ 10.1007 / \mathrm{bf02289364}$

Feldt, L. S., Woodruff, D. J., \& Salih, F. A. (1987). Statistical inference for Coefficient Alpha. Applied Psychological Measurement, 11(1), 93-103. https://doi. org/10.1177/014662168701100107

Freiberg-Hoffmann, A., \& Fernández-Liporace, M. M. (2013). Cuestionario Honey-Alonso de Estilos de Aprendizaje: Análisis de sus propiedades psicométricas en estudiantes universitarios. Summa Psicológica UST, 10(1), 103-117. https://doi.org/10.18774/448x.2013.10.41

Freiberg-Hoffmann, A., \& Fernández-Liporace, M. M. (2016). Enfoques de aprendizaje en estudiantes universitarios argentinos según el R-SPQ-2F: Análisis de sus propiedades psicométricas. Revista Colombiana de Psicología, 25(2), 307-329. https://doi.org/10.15446/rcp.v25n2.51874

Freiberg-Hoffmann, A., Berenguer, D., Fernández-Liporace, M., \& Ledesma, R. (2017). Estilos, estrategias y enfoques de aprendizaje en estudiantes universitarios de Buenos Aires. Revista Psicodebate, 17(1), 9-34. https://doi.org/10.18682/ pd.v17i1.626

Grasha, A., \& Riechmann, S. W. (1975). Student Learning Styles Questionnaire. University of Cincinnati Faculty Resource Center.

Gutiérrez-Tapias, M. (2018). Estilos de aprendizaje, estrategias para enseñar. Su relación con el desarrollo emocional 
y aprender a aprender. Tendencias Pedagógicas, 31, 83-96. https://doi.org/10.15366/tp2018.31.004

Hahs-Vaughn, D. L. (2017). Applied multivariate statistical concepts. Routledge.

Halili, S. H., Sulaiman, S., Sulaiman, H., \& Razak, R. (2019). Exploring students' learning styles in using mobile flipped classroom. International and Multidisciplinary Journal of Social Sciences, 8(2), 105-125. https://doi.org/10.17583/ rimcis. 2019.4070

Holgado-Tello, F. P., Morata-Ramírez, M. Á., \& BarberoGarcía, M. I. (2018). Confirmatory Factor Analysis of Ordinal Variables: A Simulation Study Comparing the Main Estimation Methods. Avances en Psicología Latinoamericana, 36(3), 601. https://doi.org/10.12804/re vistas.urosario.edu.co/apl/a.4932

Honey, P., \& Mumford, A. (1986). The manual of learning styles. P. Honey, Ardingly House.

Irwin, P., \& Hughes, D. J. (2018). Test development. En P. Irwin, T. Booth \& D. J. Hughes (Eds.), The Wiley handbook of psychometric testing (Vol I, pp. 3-47). Wiley.

Jackson, C. J. (2002). Learning styles and it measurement: An applied neuropsychological model of learning for business and education. Cymeon.

Jackson, D. L., Gillaspy, J. A., \& Purc-Stephenson, R. (2009). Reporting practices in confirmatory factor analysis: An overview and some recommendations. Psychological Methods, 14(1), 6-23. https://doi.org/10.1037/a0014694

Jak, S. (2014). Testing strong factorial invariance using threelevel structural equation modeling. Frontiers in Psychology, 5, 1-7. https://doi.org/10.3389/fpsyg.2014.00745

Jiraporncharoen, W., Angkurawaranon, C., Chockjamsai, M., Deesomchok, A., \& Euathrongchit, J. (2015). Learning styles and academic achievement among undergraduate medical students in Thailand. Journal of Educatoinal Evaluation for Health Professions, 12(38), 1-7. https:/doi. org/10.3352/jeehp.2015.12.38

Juárez-Lugo, C., Rodríguez-Hernández, G., \& Luna-Montijo, E. (2012). El cuestionario de estilos de aprendizaje CHAEA y la escala de estrategias de aprendizaje ACRA como herramienta potencial para la tutoría académica. Revista de Estilos de Aprendizaje, 10, 1-31. http://revistaestilosdeapren dizaje.com/article/view/965/1673

Keefe, J. (1982). Assesing student learning styles. An Overview. ERIC.

Koğar, H., \& Yilmaz-Koğar, E. (2015). Comparison of different estimation methods for categorical and ordinal data in confirmatory factor analysis. Journal of Measurement and Evaluation in Education and Psychology, 6(2), 351-364. https://doi.org/10.21031/epod.94857

Kolb, D. (1976). Learning Style Inventory: Technical Manual. McBer and Company.

Kolb, D. (1984). Experiential learning: experience as the source of learning and development. Prentice Hall, Inc.
Kolb, A., \& Kolb, D. (2005). The Kolb learning style inventory (version 3.1), 2005 technical specifications. HayGroup. www.whitewater-rescue.com/support/pagepics/lsitechman ual.pdf

Li, C. H. (2015). Confirmatory factor analysis with ordinal data: Comparing robust maximum likelihood and diagonally weighted least squares. Behavior Research Methods, 48(3), 936-949. https://doi.org/:10.3758/s13428-015-0619-7

Lopes da Silveira, P. A. (2013). Análisis multivariante de la relación entre estilos/estrategias de aprendizaje e inteligencia emocional, en alumnos de educación superior (Tesis doctoral). Departamento de Estadística, Universidad de Salamanca, España. https://repositorio. ipcb.pt/bitstream/10400.11/1775/1/Tesis_Paulo\%20 Silveira_\%C3\%9Altima\%20Versi\%C3\%B3n_8\%20 Marzo.pdf

Mozaffari, H. R., Janatolmakan, M., Sharifi, R., Ghandinejad, F., Andayeshgar, B., \& Khatony, A. (2020). The relationship between the VARK learning styles and academic achievement in Dental Students. Advances in Medical Education and Practice, 11, 15-19. https://doi.org/10.2147/amep. s235002

Mulaik, S. A., James, L. R., Van-Alstine, J., Bennet, N., Lind, S., \& Stilwell, C. D. (1989). Evaluation of Goodness-of-Fit Indices for Structural Equation Models. Psychological Bulletin, 105(3), 430-45. https://doi. org/10.1037//0033-2909.105.3.430

Myers, I. B., \& Myers, P. B. (1980). Manual: A guide to use of the Myers-Briggs Tipe Indicator. Consulting Psychologist Press.

Olanipekun, T., Effoe, V., Bakinde, N., Bradley, C., Ivonye, C., \& Harris, R. (2020). Learning Styles of Internal Medicine Residents and Association With the In-Training Examination Performance. Journal of the National Medical Association, 112(1), 44-51. https://doi.org/10.1016/j.jnma.2019.12.002

Orellana, N., Bo, R., Belloch, C., \& Aliaga, F. (2002). Estilos de aprendizaje y utilización de las TIC en la enseñanza superior. Actas de la Conferencia Internacional sobre Educación, Formación y Nuevas Tecnologías. Virtual Educa 2004, Valencia 117. http://www.uv.es/ bellochc/ doc\%20UTE/VE2002_117.pdf

Ponce-Cumbreras, J., \& Gamarra-Bustillos, C. (2015). Estilos de aprendizaje y rendimiento académico en estudiantes de la Universidad de María Auxiliadora. Ágora Revista Cientifica, 2(1), 105-111. https://doi.org/10.21679/arc. v2i1.24

Prieto-Loureiro, G. (2019). Identificación de los estilos de aprendizaje para el aprendizaje de contenidos clínicos en estudiantes de psicología, a través del Cuestionario HoneyAlonso (CHAEA). Alternativas Cubanas en Psicología, 7(19), 122-134. https://www.acupsi.org/articulo/254/iden tificacin-de-los-estilos-de-aprendizaje-para-el-aprendizajede-contenidos-clnicos-en-estudiantes-de-psicologa-a-travsdel-cuestionario-horney-alonso-chaea.html 
Ranganathan, P., \& Aggarwal, R. (2016). Common pitfalls in statistical analysis: The use of correlation techniques. Perspectives in Clinical Research, 7(4), 187-190. https:// doi.org/10.4103/2229-3485.192046

Renzulli, J. S., \& Smith, L. H. (1978). Learning Styles Inventory: A measure of student preference for instructional techniques. Creative Learning Press.

Rezler, A. G., \& Rezmovic, V. (1981). The Learning Preference Inventory. Journal of Allied Health, 19(1), 28-34. https:// www.ncbi.nlm.nih.gov/pubmed/7228814

Rodríguez, H. de J. D., Limón, J. A., Pisfil, M. L., Torres, D. V., \& Exume, J. C.. (2015). Estilos de aprendizaje: un estudio diagnóstico en el centro universitario de ciencias económico-administrativas de la U de G. Revista de La Educación Superior, 44(175), 121-140. https://doi. org/10.1016/j.resu.2015.09.005

Rodríguez-Gómez, J. (2006). Modelo de asociación entre los enfoques y estilos de aprendizaje en estudiantes universitarios del estado de Nuevo León (Tesis doctoral). Facultad de Educación, Universidad de Montemorelos, México. http://dspace.biblioteca. um.edu.mx/xmlui/bitstream/handle/20.500.11972/852/ Tesis\%20Jaime\%20Rodr\%C3\%8Cguez\%20Gomez. pdf? sequence $=1 \&$ isAllowed $=y$

Rojas, L., Rojas, G., \& Brizuela, A. (2018). The use of measurement invariance with dichotomous variables as evidence of validity. Evaluar, 18(2), 45-58. https://doi. org/10.35670/1667-4545.v18.n2.20807

Schmeck, R. R., Ribich, F. D., \& Ramanaiah, N. (1977). Development of a self-report inventory for assessing individual differences in learning processes. Applied Psychological Measurement 1(3), 413-431. https://doi. org/10.1177/014662167700100310
Schumacker, R., \& Lomax, R. (2016). A beginner's guide to structural equation modeling. Routledge.

Silva-Falchetti, E. (2009). Estilos de aprendizagem em universitários brasileiros. Estabelecimento de perfis por titulação. Tradução, adaptação e análise do CHAEA (Tesis doctoral). Univeridad de León, España. https://buleria.unileon. es/xmlui/bitstream/handle/10612/999/2009SILVA\%20 FALCHETTI\%2c\%20EDNA.pdf?sequence $=1$

Vermunt, J. D. (1998). The regulation of constructive learning process. British Journal of Educational Psychology, 68, 149-171. https://doi.org/10.1111/j.2044-8279.1998. tb01281.x

Villalba, A. B. (2015). Estilos de aprendizaje en alumnos universitarios de profesorado en Biología y licenciatura en Biodiversidad. Revista Estilos de Aprendizaje, 8(16), 79-100. http://revistaestilosdeaprendizaje.com/article/ view/1018

Wilkinson, T., Boohan, M., \& Stevenson, M. (2014). Does learning style influence academic performance in different form of assessment. Journal of Anatomy, 224(3), 304-308. https://doi.org/10.1111/joa.12126

Witkin, H. A., Oltman, P. K., Raskin, E., \& Karp, S. A. (1971). Group Embedded Figures Test manual. Consulting Psychology Press.

Yuan, K., Wu, R., \& Bentler, P. (2011). Ridge structural equation modeling with correlation matrices for ordinal and continuous data. British Journal of Mathematical and Statistical Psychology, 64(1), 107-133. https://doi. org/10.1348/000711010X497442 